\title{
Article \\ Factors Associated with Acute Community-Acquired Pyelonephritis Caused by Extended-Spectrum $\beta$-Lactamase-Producing Escherichia coli
}

\author{
Mónica Romero Nieto ${ }^{1,2, *}$, Sara Maestre Verdú ${ }^{1}$, Vicente Gil ${ }^{2,3}$, Carlos Pérez Barba ${ }^{1,2}$, \\ Jose Antonio Quesada Rico ${ }^{2}$ and Reyes Pascual Pérez ${ }^{1,2, *}$ \\ 1 Department of Internal Medicine, Elda General University Hospital, Elda/Sax, Road s/n, \\ 03600 Elda, Alicante, Spain; saramaver@gmail.com (S.M.V.); cperezb@coma.es (C.P.B.) \\ 2 Department of Clinical Medicine, Elche Miguel Hernández University, 03550 San Juan, Alicante, Spain; \\ vte.gil@gmail.com (V.G.); jquesada@umh.es (J.A.Q.R.) \\ 3 Research Unit, Elda General University Hospital, 03600 Elda, Alicante, Spain \\ * Correspondence: monica.romeron@umh.es (M.R.N.); mr.pascual@umh.es (R.P.P.)
}

check for updates

Citation: Romero Nieto, M.; Maestre Verdú, S.; Gil, V.; Pérez Barba, C.; Quesada Rico, J.A.; Pascual Pérez, R. Factors Associated with Acute Community-Acquired Pyelonephritis Caused by Extended-Spectrum $\beta$-Lactamase-Producing Escherichia coli. J. Clin. Med. 2021, 10, 5192. https://doi.org/10.3390/jcm10215192

Academic Editor: Jonathan Barratt

Received: 9 October 2021

Accepted: 5 November 2021

Published: 7 November 2021

Publisher's Note: MDPI stays neutral with regard to jurisdictional claims in published maps and institutional affiliations.

Copyright: (C) 2021 by the authors. Licensee MDPI, Basel, Switzerland. This article is an open access article distributed under the terms and conditions of the Creative Commons Attribution (CC BY) license (https:// creativecommons.org/licenses/by/ $4.0 /)$.
Abstract: This study aimed to identify the factors associated with the presence of extended-spectrum B-lactamase-(ESBL) in patients with acute community-acquired pyelonephritis (APN) caused by Escherechia coli (E. coli), with a view of optimising empirical antibiotic therapy in this context. We performed a retrospective analysis of patients with community-acquired APN and confirmed E. coli infection, collecting data related to demographic characteristics, comorbidities, and treatment. The associations of these factors with the presence of ESBL were quantified by fitting multivariate logistic models. Goodness-of-fit and predictive performance were measured using the ROC curve. We included 367 patients of which 51 presented with ESBL, of whom 90.1\% had uncomplicated APN, $56.1 \%$ were women aged $\leq 55$ years, $33.5 \%$ had at least one mild comorbidity, and $12 \%$ had recently taken antibiotics. The prevalence of ESBL-producing E. coli was $13 \%$. In the multivariate analysis, the factors independently associated with ESBL were male sex (OR 2.296; 95\% CI 1.043-5.055), smoking (OR 4.846, 95\% CI 2.376-9.882), hypertension (OR 3.342, 95\% CI 1.423-7.852), urinary incontinence (OR 2.291, 95\% CI 0.689-7.618) and recurrent urinary tract infections (OR 4.673, 95\% CI 2.271-9.614). The area under the ROC curve was 0.802 (IC 95\% 0.7307-0.8736), meaning our model can correctly classify an individual with ESBL-producing $E$. coli infection in $80.2 \%$ of cases.

Keywords: acute pyelonephritis; Escherichia coli; extended-spectrum ß-lactamase

\section{Introduction}

Acute pyelonephritis (APN) accounts for a large proportion of community-acquired and hospital-acquired urinary tract infections [1]. Though less common than cystitis, APN is associated with significant morbidity and can lead to serious complications, including death. In the USA, it kills around 4000 people each year [2,3]. Evidence regarding the aetiology of this infection is mostly extrapolated from studies on cystitis [4]. European studies have reported an increase in antibiotic resistance in Gram-negative bacilli, especially Escherichia coli (E. coli), with frequent cross-resistance to fluoroquinolones, and B-lactamaseproducing strains $[5,6]$. The increasing presence of extended-spectrum ß-lactamase (ESBL)producing E. coli strains in urine culture isolates of people with community-acquired APN is serious problem that leads to considerable use of healthcare resources [7-9]. Population ageing, increasing immunosuppression and the growing frequency of urinary catheterisation, among other factors, have given rise to multidrug-resistant microorganisms [10]. Previous studies have identified the following risk factors for developing B-lactamaseproducing strains: age over 55 years, prior use of antibiotics, prior urinary tract infections (UTIs), and diabetes mellitus [11,12]. Inadequate antibiotic therapy has been associated 
with increased morbidity $[13,14]$. Moreover, different studies have shown a wide variability in aetiology, depending on the place of acquisition, age, and comorbidities $[2,15,16]$.

It is therefore crucial to regularly review APN-causing microorganisms and their sensitivity to antibiotics $[17,18]$, and to identify the characteristics and factors associated with antimicrobial resistance [19] The literature contains very few studies on E. coli resistance in community-acquired APN in Spain or in the whole of Europe, and associated factors are rarely examined. In this study, we aimed to determine the prevalence of ESBL-producing E. coli in cases of community-acquired APN caused by E. coli, identify the factors associated with the presence of these strains and to use this information to design a explicative model for use in the determination of empirical antibiotic therapy regimens.

\section{Materials and Methods}

We conducted a cross-sectional study, analysing cases of community-acquired APN caused by E. coli that required hospital admission in Elda General University Hospital (Spain), which serves a population of 194,000 inhabitants (with 400 hospital beds, which has an infectious Disease Unit integrated into the internal medicine service, with 15 beds in its care). The study period spanned from 1 January 2012 to 31 June 2018. We included patients aged 14 and older in whom E. coli was isolated in urine or blood cultures. We excluded patients with no cultures, with negative results, in whom other microorganisms were isolated without $E$. coli, and who had incomplete information. We also excluded all cases of APN acquired in a care setting.

We searched for the APN diagnostic code in all electronic hospital discharge records created during the study period. After applying the inclusion criteria, we collected data related to demographic characteristics, comorbidities, Charlson comorbidity index, urinary pathology, urinary catheterisation, prior use of antibiotics, length of hospital stay, antimicrobial sensitivity, and prescribed empirical antibiotic therapy.

We applied the following definitions during data collection:

APN: a urinary tract infection infecting the upper urinary tract (renal pelvis and kidney parenchyma), usually causing fever, flank pain, nausea, vomiting, and clinical features of lower tract infection (frequent urination and, more rarely, tenesmus or incontinence).

Complicated APN: APN that worsens and leads to acute focal nephritis, renal corticomedullary abscess, perirenal abscess, papillary necrosis, or emphysematous pyelonephritis.

First admission: first time the patient was admitted with a primary diagnosis of APN.

B-lactamase: an enzyme, produced by some bacteria, that confers resistance to $B$ lactam antibiotics-such as penicillins, cephalosporins, monobactams and carbapenems (carbapenemases) - by hydrolysing the $§$-lactam ring and generating a derivative without antimicrobial activity.

ESBLs: enzymes derived mainly from TEM and SHV-type enzymes (also described in CTX and OXA) and that can hydrolyse penicillins, broad-spectrum cephalosporins, and monobactams.

Positive urine culture result: $>10^{4}$ colony-forming units (CFU) of E. coli.

Positive blood culture result: E. coli isolated in at least one blood culture.

Readmission: admission for the same reason within 30 days of discharge.

Relapse: recurrence of the disease after a period of remission or apparent recovery.

Sepsis: defined according to the 2012 Surviving Sepsis Campaign criteria [20], as the study period began in 2012 .

Assuming a worst-case scenario of $50 \%$ prevalence, the sample size needed to estimate the proportion of ESBL-producing E. coli with a $95 \%$ confidence interval and a precision of $5 \%$ was 385 patients.

We performed a descriptive analysis by calculating absolute and relative frequencies for the categorical variables, and ranges, means and standard deviations for the quantitative variables. We analysed the factors associated with the presence of ESBL using contingency tables, applying a chi-squared test for the categorical variables, and comparing means with a Student's $t$-test for the quantitative variables. 
To quantify the association of each variable with the presence of ESBL, we fitted multivariate logistic models. Odds ratios (ORs) were calculated together with their 95\% confidence intervals. Variables were selected in a stepwise procedure based on the Akaike Information Criterion. Goodness-of-fit and predictive performance were measured using the ROC curve.

For each cutoff value for the probability of presenting with ESBL, obtained through the multivariate logistic regression equation, we calculated validity indicators (sensitivity and specificity), predictive values and likelihood ratios. For all these indicators, we calculated 95\% confidence intervals. To interpret the cutoff values and thus confirm or reject the diagnosis of APN caused by ESBL-producing E. coli, we applied evidence-based medicine criteria.

All analyses were performed with SPSS version 25 and $\mathrm{R}$ version 3.5.1.

\section{Results}

We reviewed 724 cases with a diagnosis of APN on discharge, of which 367 met the inclusion criteria of our study.

Figure 1 shows the reasons for excluding the remaining 357 patients.

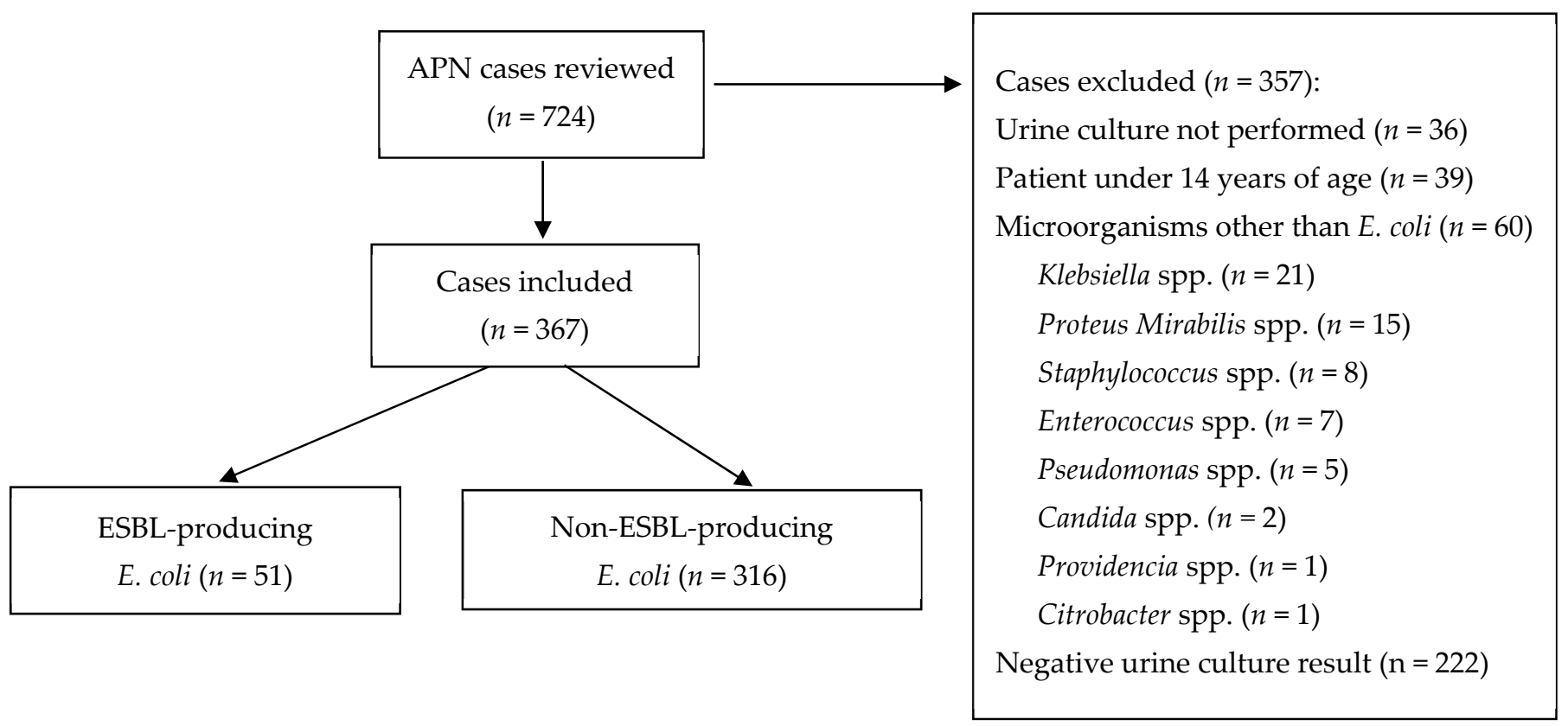

Figure 1. APN cases analysed, included, and excluded, with reasons for exclusion.

Table 1 shows the baseline characteristics of the sample.

Most patients were women aged under 55 years $(56.1 \%)$. One third of patients $(33.5 \%)$ had at least one mild comorbidity, and $12 \%$ had taken antibiotics in the last three months.

Most patients (62.7\%) were admitted to the short stay unit. Table 2 shows which departments patients were admitted to.

Most cases of APN were uncomplicated (90.1\%). Eighteen patients (4.9\%) developed sepsis and $99(27 \%)$ had acute kidney injury. Blood cultures were performed in 247 patients $(67.3 \%)$, and in $7.3 \%$ of all patients, ESBL-producing E. coli was isolated in blood cultures. The prevalence of ESBL-producing E. coli in cases of APN caused by E. coli was $13.9 \%$ $(n=51 ; 95 \%$ CI 10.4-17.4). Most cases were in women aged over 75 years, with statistically significant differences (Table 3). The prevalence of ESBL increases with age in female patients, reaching $26.9 \%$ in women over 75 years (Table 3 ). 
Table 1. Baseline characteristics of patients included in the study.

\begin{tabular}{|c|c|c|}
\hline Variable & $n$ & $\%$ \\
\hline \multicolumn{3}{|l|}{ Year of admission } \\
\hline 2012 & 41 & $(11.2 \%)$ \\
\hline 2013 & 62 & $(16.9 \%)$ \\
\hline 2014 & 60 & $(16.3 \%)$ \\
\hline 2015 & 62 & $(16.9 \%)$ \\
\hline 2016 & 61 & $(16.6 \%)$ \\
\hline 2017 & 60 & $(16.3 \%)$ \\
\hline 2018 * & 21 & $(5.7 \%)$ \\
\hline \multicolumn{3}{|l|}{ Age } \\
\hline$<55$ years & 206 & $(56.1 \%)$ \\
\hline $55-74$ years & 93 & $(25.3 \%)$ \\
\hline$\leq 75$ years & 68 & $(18.5 \%)$ \\
\hline \multicolumn{3}{|l|}{ Sex } \\
\hline female & 293 & $(79.8 \%)$ \\
\hline male & 74 & $(20.2 \%)$ \\
\hline \multicolumn{3}{|l|}{ Type of admission } \\
\hline First admission & 359 & $(97.8 \%)$ \\
\hline Readmission & 8 & $(2.2 \%)$ \\
\hline Smoker (yes) & 151 & $(41.1 \%)$ \\
\hline Alcohol (yes) & 40 & $(10.9 \%)$ \\
\hline General surgery (yes) & 237 & $(64.6 \%)$ \\
\hline \multicolumn{3}{|l|}{ CCI (age-adjusted) } \\
\hline 0 & 176 & $(48.0 \%)$ \\
\hline 1-2 (mild) & 123 & $(33.5 \%)$ \\
\hline$\leq 3$ (severe) & 68 & $(18.5 \%)$ \\
\hline Hypertension (yes) & 131 & $(35.8 \%)$ \\
\hline Diabetes mellitus (yes) & 62 & $(16.9 \%)$ \\
\hline Dependent ${ }^{* *}$ (yes) & 25 & $(6.8 \%)$ \\
\hline Solid tumour (yes) & 19 & $(5.2 \%)$ \\
\hline Cardiovascular disease (yes) & 53 & $(14.4 \%)$ \\
\hline Chronic kidney disease (yes) & 20 & $(5.4 \%)$ \\
\hline Urinary incontinence (yes) & 19 & $(5.2 \%)$ \\
\hline Use of antibiotics in last 3 months (yes) & 44 & $(12.0 \%)$ \\
\hline Recurrent urinary tract infections (yes) & 78 & $(21.3 \%)$ \\
\hline
\end{tabular}

* APN cases included from 1 January 2018 to 31 June 2018. ${ }^{* *}$ Dependent (the patient need help for basic daily routines.

Table 2. Departments where APN patients were admitted.

\begin{tabular}{ccc}
\hline Department & $\boldsymbol{n}$ & $\mathbf{( \% )}$ \\
\hline Short stay unit & 230 & $(62.7)$ \\
Urology & 36 & $(9.8)$ \\
Internal medicine & 25 & $(6.8)$ \\
Emergency department & 16 & $(4.4)$ \\
Other & 14 & $(3.8)$ \\
\hline
\end{tabular}

Table 4 shows the multivariate logistic model for the presence of ESBL. Six variables (age, sex, smoking status, hypertension, urinary incontinence, recurrent UTIs) entered the model, giving a significant result $(\mathrm{X} 266.4 ; p<0.001)$. With the exception of age $(p=0.649)$ and urinary incontinence $(p=1.076)$, which acted as potential confounders, all variables showed statistical significance $(p<0.05)$, with ORs associated with increased likelihood of presence of ESBL. The variables with the highest odds radios were smoking status $(\mathrm{OR}=4.846)$ and recurrent UTIs $(\mathrm{OR}=4.673)$. 
Table 3. Prevalence of extended-spectrum ß-lactamase (ESBL)-producing Escherichia coli (E. coli) in patients with acute pyelonephritis caused by E. coli, by sex and age.

\begin{tabular}{cccccc}
\hline Scheme & \multicolumn{2}{c}{ Non-ESBL E. coli } & \multicolumn{2}{c}{ ESBL E. coli } & $p$ Value \\
\hline Women aged $<55$ years & $\boldsymbol{n}$ & $\%$ & $\boldsymbol{n}$ & $\%$ & \\
Women aged 55-74 years & 163 & $91.6 \%$ & 15 & $8.4 \%$ & $<0.001$ \\
Women aged $\geq 75$ years & 36 & $88.9 \%$ & 7 & $11.1 \%$ & \\
Men aged $<55$ years & 25 & $73.1 \%$ & 14 & $26.9 \%$ & \\
Men aged 55-74 years & 21 & $70.3 \%$ & 3 & $10.7 \%$ & 0.186 \\
Men aged $\geq 75$ years & 13 & $81.2 \%$ & 3 & $30.0 \%$ & \\
\hline
\end{tabular}

Table 4. Multivariate logistic model for the presence of extended-spectrum ß-lactamases.

\begin{tabular}{ccccc}
\hline & Coefficient & OR & $\mathbf{9 5 \%}$ CI & $p$ Value \\
\hline Intercept & -3.7101 & & & \\
Age & -0.0049 & 0.995 & $(0.974-1.017)$ & 0.649 \\
Sex (man) & 0.8309 & 2.296 & $(1.043-5.055)$ & 0.039 \\
Smoker (yes) & 1.5781 & 4.846 & $(2.376-9.882)$ & $<0.001$ \\
Hypertension (yes) & 1.2066 & 3.342 & $(1.423-7.852)$ & 0.006 \\
Urinary incontinence (yes) & 0.8290 & 2.291 & $(0.689-7.618)$ & 0.176 \\
Recurrent UTIs (yes) & 1.5417 & 4.673 & $(2.271-9.614)$ & $<0.001$ \\
\hline
\end{tabular}

UTI: urinary tract infection.

Figure 2 shows the ROC curve, with an area under the curve of 0.802 (95\% CI 0.7307-0.8736). The multivariate logistic regression equation was as follows:

$$
\text { Probability of having ESBL }=\frac{\mathrm{A}}{1+\mathrm{A}}
$$

where $\mathrm{A}=\exp [-3.7101-0.0049$ AGE + 0.8309 SEX + 1.5781 SMOKING + 1.2066 HYPERTENSION + 0.8290 URINARY INCONTINENCE + 1.5417 RECURRENT UTIs], and where the items in the equation were defined as follows: age (years), sex ( 1 men, 0 woman), smoking ( 1 if smoker, 0 if nonsmoker or ex-smoker), hypertension ( 1 if yes, 0 if no), urinary incontinence ( 1 if yes, 0 if no), recurrent UTIs ( 1 if yes, 0 if no).

Tables 5 and 6 show the different cutoffs for the probability of presenting with ESBL according to the model, with the following summary measures: sensitivity and specificity with 95\% confidence intervals, Youden index, accuracy, positive and negative predictive values with $95 \%$ confidence intervals, and positive and negative likelihood ratios with $95 \%$ confidence intervals. 


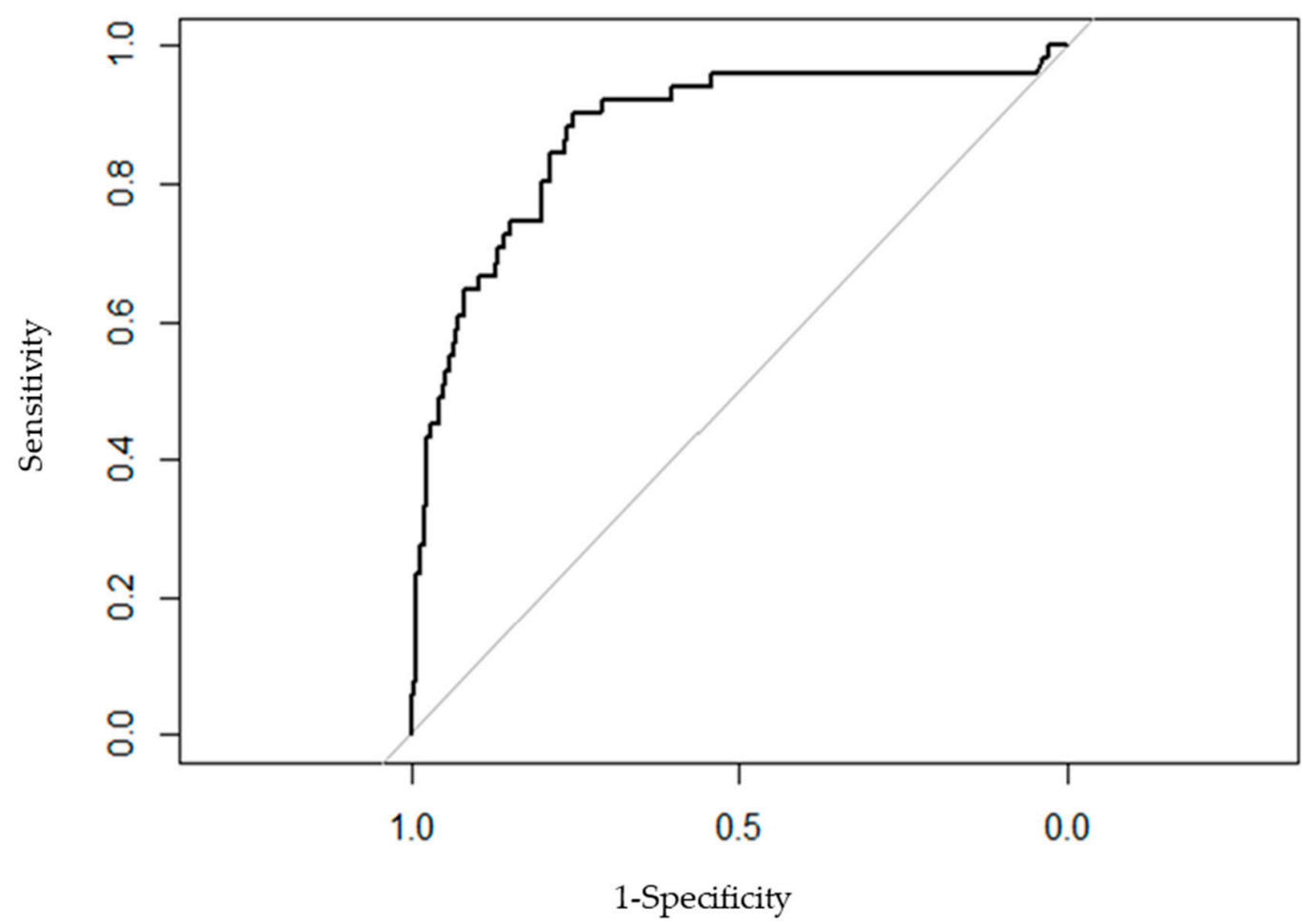

Figure 2. ROC curve of the adjusted multivariate model.

Table 5. Sensitivity and specificity with $95 \%$ confidence intervals (CI), Youden index, and accuracy for the different cutoff values.

\begin{tabular}{ccccc}
\hline Cutoff & Sensitivity $\mathbf{( 9 5 \%}$ CI) & Specificity $\mathbf{( 9 5 \%}$ CI) & Youden Index & Accuracy \\
\hline 0.05 & $94.10(87.6-100.6)$ & $34.90(29.6-40.2)$ & 0.290 & 43.20 \\
0.10 & $70.60(58.1-83.1)$ & $72.40(67.5-77.3)$ & 0.430 & 72.10 \\
0.15 & $68.60(55.9-81.3)$ & $78.70(74.2-83.2)$ & 0.473 & 77.30 \\
0.20 & $62.70(49.4-76.0)$ & $82.90(78.7-87.1)$ & 0.456 & 80.10 \\
0.25 & $52.90(39.2-66.6)$ & $91.10(88.0-94.2)$ & 0.440 & 85.80 \\
0.30 & $51.00(37.3-64.7)$ & $92.70(89.8-95.6)$ & 0.437 & 86.90 \\
0.35 & $47.10(33.4-60.8)$ & $94.30(91.7-96.9)$ & 0.414 & 87.70 \\
0.40 & $35.30(22.2-48.4)$ & $96.80(94.9-98.7)$ & 0.321 & 88.30 \\
0.45 & $33.30(20.4-46.2)$ & $97.50(95.8-99.2)$ & 0.308 & 88.50 \\
0.50 & $33.30(20.4-46.2)$ & $98.40(97.0-99.8)$ & 0.317 & 89.30 \\
0.55 & $27.50(15.2-39.8)$ & $98.40(97.0-99.8)$ & 0.259 & 88.50 \\
0.60 & $15.70(5.7-25.7)$ & $99.00(97.9-100.1)$ & 0.147 & 87.40 \\
0.65 & $13.70(4.3-23.1)$ & $99.00(97.9-100.1)$ & 0.127 & 87.20 \\
0.70 & $13.70(4.3-23.1)$ & $99.00(97.9-100.1)$ & 0.127 & 87.20 \\
\hline
\end{tabular}

Facing a new patient, the multivariate logistic model shows the probability of the appearance of ESBL when replacing the values for the new patient with the variables in the multivariate model.

This probability takes values between 0 and 1 . In order to generate a prediction about the possibility that the new patient will present with ESBL, a probability cutoff point is needed. Starting from that point, we will classify the new patient by ESBL presence.

A standard way to fix the cutoff point is to take 0.5 , but also some predictive values, such as the sensibility and specificity, can be calculated for all the cutoff points between 0 and 1 . In this way an optimal cut-off point can be chosen.

The optimal cutoff value corresponds to a $15 \%$ probability of having ESBL, with a sensitivity of $68.6 \%$ and a specificity of $78.7 \%$. The positive likelihood ratios (LR+) that produce marked increases in the certainty of a positive ESBL-APN diagnosis (LR+ $>10)$ correspond to a probability of $40 \%$ or higher. The negative likelihood ratio (LR-) that 
produces moderate to marked increases in the certainty of a negative ESBL-APN diagnosis (LR - around 0.1 ) corresponds to a probability of 5\% (Table 6).

Table 6. Predictive values and likelihood ratios with 95\% confidence intervals (CI).

\begin{tabular}{ccccc}
\hline Cutoff & PPV (95\% CI) & NPV (95\% CI) & LR+ (95\% CI) & LR- (95\% CI) \\
\hline 0.05 & $19.00(14.2-23.8)$ & $97.30(94.3-100.3)$ & $1.45(1.3-1.6)$ & $0.17(0.1-0.5)$ \\
0.10 & $29.30(21.3-37.3)$ & $93.80(90.8-96.8)$ & $2.56(2.1-3.1)$ & $0.41(0.3-0.6)$ \\
0.15 & $34.30(25.1-43.5)$ & $93.90(91.0-96.8)$ & $3.22(2.6-4.0)$ & $0.40(0.3-0.6)$ \\
0.20 & $37.20(27.0-47.4)$ & $93.20(90.3-96.1)$ & $3.67(2.8-4.7)$ & $0.45(0.3-0.6)$ \\
0.25 & $49.10(35.9-62.3)$ & $92.30(89.3-95.3)$ & $5.94(4.1-8.5)$ & $0.52(0.4-0.7)$ \\
0.30 & $53.10(39.1-67.1)$ & $92.10(89.1-95.1)$ & $6.99(4.7-10.4)$ & $0.53(0.4-0.7)$ \\
0.35 & $57.10(42.1-72.1)$ & $91.70(88.7-94.7)$ & $8.26(5.2-13.0)$ & $0.56(0.4-0.7)$ \\
0.40 & $64.30(46.6-82.0)$ & $90.20(87.0-93.4)$ & $11.03(6.0-20.4)$ & $0.67(0.5-0.8)$ \\
0.45 & $68.00(49.7-86.3)$ & $90.00(86.8-93.2)$ & $13.32(6.7-26.6)$ & $0.68(0.6-0.8)$ \\
0.50 & $77.30(59.8-94.8)$ & $90.10(86.9-93.3)$ & $20.81(8.7-49.9)$ & $0.68(0.6-0.8)$ \\
0.55 & $73.70(53.9-93.5)$ & $89.30(86.0-92.6)$ & $17.19(7.2-41.2)$ & $0.74(0.6-0.9)$ \\
0.60 & $72.70(46.4-99.0)$ & $87.90(84.5-91.3)$ & $15.70(5.1-48.6)$ & $0.85(0.8-1.0)$ \\
0.65 & $70.00(41.6-98.4)$ & $87.60(84.2-91.0)$ & $13.70(4.4-42.4)$ & $0.87(0.8-1.0)$ \\
0.70 & $70.00(41.6-98.4)$ & $87.60(84.2-91.0)$ & $13.70(4.4-42.4)$ & $0.87(0.8-1.0)$ \\
\hline
\end{tabular}

PPV: positive predictive value; NPV: negative predictive value; LR+: positive likelihood ratio; LR-: negative likelihood ratio.

\section{Discussion}

In our study, the prevalence of ESBL-producing E. coli in cases of community-acquired APN caused by E. coli was 13.9\%, considering that we studied 367 APN of which 51 were ESBL. Male sex, smoking, hypertension, urinary incontinence, and recurrent UTIs were associated with the presence of ESBL-producing E. coli, and the model applied can correctly predict this outcome in $80.2 \%$ of cases.

Our prevalence is similar to the $12 \%$ prevalence observed by Talan et al. [21]. Data on APN are scarce: most publications provide global results from all isolates without specific reference to APN. In a recent study from Korea, ESBL-producing E. coli was isolated in up to $29 \%$ of cases of community-acquired APN [22,23]. In Spain, ESBL-producing strains of E. coli and Klebsiella pneumoniae have caused an increase in the prevalence of multidrug-resistant isolates of these bacteria in recent years, both in hospitals [10] and in the community [24]. In Europe, the prevalence of B-lactamase-producing strains in community-acquired urinary tract infections is higher than in the USA, but lower that in Asia or South America [25].

Our study shows little fluctuation in prevalence since 2012, which is consistent with the steady $10 \%$ prevalence reported in another recent paper [11]. However, other studies have found an upward trend [25].

Most of our patients were women aged under 55 years; $42 \%$ had at least one comorbidity, and a high percentage were smokers. Almost one third had hypertension and one fifth had a history of urinary tract infections. Most patients were admitted to the short stay unit, corresponding to the normal length of a stay for uncomplicated APN. However, the patients admitted to the short stay unit had a lower percentage of ESBL-producing E. coli isolates than those admitted to the infectious disease unit. This shows that cases of greater complexity, in terms of clinical features and/or antibiotic therapy, tend to be admitted or transferred to specialised units.

The percentage of complicated APN in our patients (9.9\%) was lower than in previous studies, possibly because these studies included patients with hospital-acquired as well as community-acquired APN and adopted a broader definition of complicated APN [21,26].

In the multivariate analysis, age was associated with the presence of ESBL-producing E. coli, as in previous studies [11]. A case-control study by Sun Hee Park et al. showed that age, prior use of antibiotics, diabetes and recurrent UTIs were independent risk factors for developing APN caused by ESBL-producing E. coli. In our analysis, this prevalence 
increased with patient age in women only, ranging from $8 \%$ in those aged under 55 years to $26 \%$ in those aged over 75 years. The higher number of comorbidities and greater exposure to antibiotics probably contribute to the higher prevalence of this resistant strain in older people [27-30]. Although this association did not show statistical significance in the multivariate analysis, we believe age could be a relevant factor to consider when proposing empirical antibiotic therapy.

As in previous studies [12,30-32], we found that patients with a history of UTIs were more likely to have ESBL-producing E. coli, which may be related to repeated use of antibiotics favouring the selection of multidrug-resistant microorganisms.

Although hypertension was prevalent in our sample, the multivariate analysis showed it to be an independent factor. We have found no other studies with similar results. Vascular damage caused by hypertension could lead to renal ischaemia and contribute to increasing susceptibility to infection, but this would not explain the appearance of resistance. Other factors associated with hypertension (e.g., older age, diabetes or prostate problems in men) could also play a role, although none of them showed statistical significance in the univariate analysis.

Smokers often have a wider range of comorbidities, including COPD, which can lead to respiratory infections. The antibiotics prescribed to treat these infections make patients more susceptible to antibiotic resistance [30].

The ROC curve of the multivariate model had a moderate area under the curve. The area under the curve is defined as the probability of correctly classifying a pair of randomly selected APN patients, one with ESBL-producing E. coli and one without, by applying the multivariate logistic regression equation. An area under the curve of 0.802 means that $80.2 \%$ of the time a randomly selected individual from the group of patients with APN caused by ESBL-producing E. coli will have a higher risk score estimated by the multivariate model than a randomly selected individual from the group with APN caused by non-ESBL E. coli.

The model obtained from the multivariate analysis together with the probability of having ESBL-producing E. coli, calculated using the logistic equation, and the summary measures for each probability cutoff value (sensitivity, specificity, predictive values and likelihood ratios) are highly relevant for clinical practice. The cutoff value for the probability of having ESBL-producing E. coli that maximises sensitivity and specificity is 0.15 , but any other cutoff can be chosen depending on whether we are aiming for higher sensitivity (fewer false negatives) or higher specificity (fewer false positives).

In clinical practice, if a patient presents with suspected APN, the variables of the model can be measured and entered into the equation to obtain the probability of ESBL-producing E. coli infection. If we wish to classify this new patient as having or not having APN due to ESBL-producing E. coli, we can choose a probability cutoff value and use it to classify the patient according to whether their probability is higher or lower than the chosen cutoff. For each cutoff value, the clinician can establish all the epidemiological indicators.

Our study has some limitations. Firstly, it was conducted in a single hospital and the results should be corroborated before extrapolation to other contexts. As it was a retrospective study, some data may have been missing, although all the model predictors were present before the appearance of APN. Additionally, to ensure uniformity in data collection, we used the 2012 definition of sepsis, which is now considered outdated. Another limitation concerns the exclusion of APN patients who were not admitted to hospital, which may have resulted in the underreporting of cases. Unfortunately, we were unable to include these patients owing to limited availability of outpatient data.

Another limitation was that we did not have information on previous therapy received by each patient. We analysed colonisation by resistant microorganisms, but found no statistically significant association with ESBL-producing E. coli isolates in urine or blood cultures.

The main strength of our study is that it provides data on resistance in a specific infectious pathology, filling an information gap in Spain. 
Our methodology is robust, and we built an explanatory model to help clinicians choose the best empirical antibiotic therapy.

In conclusion, the prevalence ESBL-producing E. coli in patients with APN caused by $E$. coli in our study was $13 \%$ and did not vary over the years. Male sex, smoking, hypertension, urinary incontinence, and recurrent UTIs were associated with the presence of ESBL-producing E. coli in people admitted to our hospital with community-acquired APN caused by E. coli. The multivariate model could be useful in clinical practice for diagnosing APN caused by ESBL-producing E. coli with moderate discriminative capacity.

Author Contributions: Conceptualization, methodology, writing—original draft preparation, investigation, validation, writing-review and editing, resources, project administration, supervision, S.M.V., M.R.N.; conceptualization, software, investigation, writing—review, visualization, supervision V.G.; conceptualization, methodology, formal analysis, resources, writing-review, supervision, project administration C.P.B.; conceptualization, software, validation, visualization, supervision; J.A.Q.R.; methodology, software, data curation, writing - review and editing, formal analysis, writing-review, visualization, supervision, R.P.P.; conceptualization, methodology, writing-original draft preparation, investigation, validation, writing-review and editing, resources, project administration, supervision. All authors have read and agreed to the published version of the manuscript.

Funding: This research received no external funding.

Institutional Review Board Statement: The study was conducted according to the guidelines of the Declaration of Helsinki and approved by the Ethics Committee of Elda General University Hospital.

Informed Consent Statement: Not applicable.

Data Availability Statement: All of the data used for this analysis can be confirmed at any time.

Conflicts of Interest: The authors declare no conflict of interest.

\section{References}

1. Laupland, K.; Ross, T.; Pitout, J.; Church, D.; Gregson, D. Community-onset urinary tract infections: A population-based assessment. Infection 2007, 35, 150-153. [CrossRef] [PubMed]

2. Czaja, C.A.; Scholes, D.; Hooton, T.M.; Stamm, W.E. Population-based epidemiologic analysis of acute pyelonephritis. Clin. Infect. Dis. 2007, 45, 273-280. [CrossRef] [PubMed]

3. Lagu, T.; Rothberg, M.B.; Shieh, M.-S.; Pekow, P.S.; Steingrub, J.S.; Lindenauer, P.K. Hospitalizations, costs, and outcomes of severe sepsis in the United States 2003 to 2007. Crit. Care Med. 2012, 40, 754-761. [CrossRef] [PubMed]

4. Bruyère, F.; Vidoni, M.; Péan, Y.; Ruimy, J.A.; Elfassi, R. Bacteriological analysis of more than 600 febrile urinary infections managed in a community health network. Prog. Urol. 2013, 23, 890-898. [CrossRef]

5. Eurosurveillance Editorial Team. ECDC publishes 2014 surveillance data on antimicrobial resistance and antimicrobial consumption in Europe. Eurosurveillance 2015, 20, 30068.

6. Scholes, D.; Hooton, T.M.; Roberts, P.L.; Gupta, K.; Stapleton, A.E.; Stamm, W.E. Risk factors associated with acute pyelonephritis in healthy women. Ann. Intern. Med. 2005, 142, 20-27. [CrossRef]

7. Calbo, E.; Romaní, V.; Xercavins, M.; Gómez, L.; Vidal, C.G.; Quintana, S.; Vila, J.; Garau, J.J. Risk factors for communityonset urinary tract infections due to Escherichia coli harbouring extended-spectrum lactamases. J. Antimicrob. Chemother. 2006, 57, 780-783. [CrossRef] [PubMed]

8. Azap, Ö.K.; Arslan, H.; Şerefhanoğlu, K.; Çolakoğlu, Ş.; Erdoğan, H.; Timurkaynak, F.; Senger, S.S. Risk factors for extendedspectrum beta-lactamase positivity in uropathogenic Escherichia coli isolated from community-acquired urinary tract infections. Clin. Microbiol. Infect. 2010, 16, 147-151. [CrossRef]

9. Pigrau, C. Nocosomial urinary tract infections. Enferm. Infecc. Microbiol. Clin. 2013, 31, 614-624. [CrossRef]

10. Sánchez-García, J.M.; Sorlózano-Puerto, A.; Navarro-Marí, J.M.; Fernández, J.G. Evolution of the antibiotic-resistance of microorganisms causing urinary tract infections: A 4-year epidemiological surveillance study in a hospital population. Rev. Clin. Esp. 2019, 219, 116-123. [CrossRef]

11. Park, S.H.; Choi, S.-M.; Lee, D.-G.; Cho, S.-Y.; Lee, H.-J.; Choi, J.-K.; Choi, J.-H.; Yoo, J.-H. Impact of Extended-Spectrum $\beta$-Lactamase Production on Treatment Outcomes of Acute Pyelonephritis Caused by Escherichia coli in Patients without Health Care-Associated Risk Factors. Antimicrob. Agents Chemother. 2015, 59, 1962-1968. [CrossRef] [PubMed]

12. Park, K.-H.; Oh, W.S.; Kim, E.S.; Park, S.W.; Hur, J.-A.; Kim, Y.K.; Moon, C.; Lee, J.H.; Lee, C.-S.; Kim, B.-N. Factors associated with ciprofloxacin- and cefotaxime-resistant Escherichia coli in women with acute pyelonephritis in the emergency department. Int. J. Infect. Dis. 2014, 23, 8-13. [CrossRef] [PubMed] 
13. Palou, J.; Pigrau, C.; Molina, I.; Ledesma, J.M.; Angulo, J. Etiology and sensitivity of uropathogens identified in uncomplicated lower urinary tract infections in women (ARESC study): Implications on empiric therapy. Med. Clin. 2011, 136, 1-7. [CrossRef] [PubMed]

14. Jeon, J.H.; Kim, K.; Han, W.D.; Song, S.H.; Park, K.U.; Rhee, J.E.; Song, K.H.; Park, W.B.; Kim, E.S.; Park, S.W.; et al. Empirical use of ciprofloxacin for acute uncomplicated pyelonephritis caused by Escherichia coli in communities where the prevalence of fluoroquinolone resistance is high. Antimicrob. Agents Chemother. 2012, 56, 3043-3046. [CrossRef]

15. Lee, S.; Song, D.Y.; Cho, S.H.; Kwon, K.T. Impact of extended-spectrum beta-lactamase on acute pyelonephritis treated with empirical ceftriaxone. Microb. Drug Resist. 2014, 20, 39-44. [CrossRef]

16. Bouchillon, S.K.; Badal, R.E.; Hoban, D.J.; Hawser, S.P. Antimicrobial Susceptibility of Inpatient Urinary Tract Isolates of GramNegative Bacilli in the United States: Results from the Study for Monitoring Antimicrobial Resistance Trends (SMART) Program: 2009-2011. Clin. Ther. 2013, 35, 872-877. [CrossRef]

17. Koningstein, M.; van der Bij, A.K.; de Kraker, M.E.A.; Monen, J.C.; Muilwijk, J.; de Greeff, S.C.; Geerlings, S.E.; van Hall, M.A.L.; on behalf of the ISIS-AR Study Group. Recommendations for the empirical treatment of complicated urinary tract infections using surveillance data on antimicrobial resistance in the Netherlands. PLoS ONE 2014, 9, e86634. [CrossRef]

18. Allocati, N.; Masulli, M.; Alexeyev, M.F.; Di Ilio, C. Escherichia coli in Europe: An Overview. Int. J. Environ. Res. Public Health 2013, 10, 6235-6254. [CrossRef]

19. Melekos, M.D.; Naber, K.G. Complicated urinary tract infections. Int. J. Antimicrob. Agents 2000, 15, 247-256. [CrossRef]

20. Dellinger, R.P.; Levy, M.M.; Rhodes, A.; Annane, D.; Gerlach, H.; Opal, S.M.; Sevransky, J.E.; Sprung, C.L.; Douglas, I.S.; Jaeschke, R.; et al. Surviving Sepsis Campaign: International Guidelines for Management of Severe Sepsis and Septic Shock, 2012. Intensiv. Care Med. 2013, 39, 165-228. [CrossRef]

21. Talan, D.A.; Takhar, S.S.; Krishnadasan, A.; Abrahamian, F.M.; Mower, W.R.; Moran, G.J.; EMERGEncy ID NET Study Group. Fluoroquinolone-Resistant and Extended-Spectrum $\beta$-Lactamase-Producing Escherichia coli Infections in Patients with Pyelonephritis, United States. Emerg. Infect. Dis. 2016, 22, 1594-1603. [CrossRef] [PubMed]

22. Hyun, M.; Lee, J.Y.; ah Kim, H.; Ryu, S.Y. Comparison of Escherichia coli and Klebsiella pneumoniae Acute Pyelonephritis in Korean Patients. Infect. Chemother. 2019, 51, 130-141. [CrossRef]

23. Kwon, K.T.; Kim, B.; Ryu, S.-Y.; Wie, S.-H.; Kim, J.; Jo, H.-U.; Park, S.Y.; Hong, K.-W.; Kim, H.I.; Kim, H.A.; et al. Changes in Clinical Characteristics of Community-Acquired Acute Pyelonephritis and Antimicrobial Resistance of Uropathogenic Escherichia coli in South Korea in the Past Decade. Antibiotics 2020, 9, 617. [CrossRef] [PubMed]

24. Arana, D.M.; Rubio, M.; Alós, J.-I. Evolution of antibiotic multiresistance in Escherichia coli and Klebsiella pneumoniae isolates from urinary tract infections: A 12-year analysis (2003-2014). Enfermedades Infecciosas y Microbiología Clínica 2017, 35, 293-298. [CrossRef] [PubMed]

25. Canton, R.; Novais, A.; Valverde, A.; Machado, E.; Peixe, L.; Baquero, F.; Coque, T.M. Prevalence and spread of ESBL-producing Enterobacteriaceae. Clin. Microbiol. Infect. 2008, 14 (Suppl. 1), 144-153. [CrossRef]

26. Talan, D.; Takhar, S.; Krishnadasan, A.; Abrahamian, F.M.; Stamm, W.E.; Moran, G.J. Prevalence and Risk Factor Analysis of Trimethoprim-Sulfamethoxazole and Fluoroquinolone-Resistant Escherichia coli Infection among Emergency Department Patients with Pyelonephritis Antibiotic Resistance in Pyelonephritis. Clin. Infect. Dis. 2008, 47, 1155-1158. [CrossRef]

27. Morosini, M.; del Campo, R. Infecciones del tracto urinario y resistencia antimicrobiana. Rev. Clín. Esp. 2019, 219, 149-150. [CrossRef] [PubMed]

28. Gupta, K.; Hooton, T.M.; Naber, K.G.; Wullt, B.; Colgan, R.; Miller, L.G.; Moran, G.J.; Nicolle, L.E.; Raz, R.; Schaeffer, A.J.; et al. International clinical practice guidelines for the treatment of acute uncomplicated cystitis and pyelonephritis in women: A 2010 update by the Infectious Diseases. Society of America and the European Society for Microbiology and Infectious Diseases. Clin. Infect. Dis. 2011, 52, e103-e120. [CrossRef]

29. Buonaiuto, V.A.; Marquez, I.; De Toro, I.; Joya, C.; Ruiz-Mesa, J.D.; Seara, R.; Plata, A.; Sobrino, B.; Palop, B.; Colmenero, J.D. Clinical and epidemiological features and prognosis of complicated pyelonephritis: A prospective observational single hospital-based study. BMC Infect. Dis. 2014, 14, 1-8. [CrossRef]

30. Lefort, A.; Panhard, X.; Clermont, O.; Woerther, P.-L.; Branger, C.; Mentré, F.; Fantin, B.; Wolff, M.; Denamur, E. Host Factors and Portal of Entry Outweigh Bacterial Determinants to Predict the Severity of Escherichia coli Bacteremia. J. Clin. Microbiol. 2010, 49, 777-783. [CrossRef]

31. Johnson, S.W.; Anderson, D.; May, D.B.; Drew, R.H. Utility of a clinical risk factor scoring model in predicting infection with extended-spectrum $\beta$-lactamase-producing enterobacteriaceae on hospital admission. Infect. Control Hosp. Epidemiol. 2013, 34, 385-392. [CrossRef] [PubMed]

32. Søraas, A.; Sundsfjord, A.; Sandven, I.; Brunborg, C.; Jenum, P.A. Risk Factors for community-acquired urinary tract infections caused by esbl-producing enterobacteriaceae-A case-control study in a low prevalence country. PLoS ONE 2013, 8, e69581. [CrossRef] [PubMed] 